\title{
Processivity of DNA repair enzymes
}

E. Dyatlova ${ }^{1 *}$, D. Zharkov ${ }^{1,2}$

${ }^{1}$ Institute of Chemical Biology and Fundamental Medicine SB RAS, Novosibirsk, Russia

${ }^{2}$ Novosibirsk State University, Novosibirsk, Russia

*e-mail: jannie.lapt@gmail.com

Key words: target search, DNA-glycosylase, DNA repair

Motivation and Aim: The problem of rapid target search in DNA is faced by transcription factors, restriction endonucleases, DNA repair enzymes and other sequence- or structurespecific DNA-binding proteins. Theoretically, the fastest target search in DNA can be achieved by combining one-dimensional diffusion along the DNA contour (processive search) and three-dimensional diffusion (distributive search). The balance between these search modes depends on many factors affecting DNA-protein interactions, such as the presence of mono- and divalent cations, competing proteins, crowding effect, etc. Presently, the mechanisms of target search are understood only for a handful of enzymes. Methods and Algorithms: We have recently developed an assay to study target search by DNA repair enzymes, based on cleavage of oligonucleotide substrate containing two targets. Thus, the distance between the targets can be precisely controlled, and any modification can be introduced into DNA. Subsequently, the probability of correlated cleavage $\left(P_{\mathrm{cc}}\right)$ is estimated, reflecting the efficiency of enzyme transfer between the specific sites. In this work, we have investigated five repair enzymes: E. coli endonuclease VIII (Nei), its human homologs NEIL1 and NEIL2, and uracil-DNA-glycosylases (UNG) from E. coli and vaccinia virus.

Results and conclusion: As expected, $P_{\mathrm{cc}}$ of all enzymes depended on the ionic strength of the solution and the presence of $\mathrm{Mg}^{2+}$. UNG from vaccinia virus was the most sensitive to these factors, raising questions about its proficiency as a suggested processivity factor of viral DNA polymerase. Nei, NEIL1 and NEIL2 showed a peak of $P_{\mathrm{cc}}$ at low but nonzero ionic strength indicating that nonpolar interactions contribute to binding of these proteins to nonspecific DNA. This conclusion was also supported by analyzing amino acid conservation in the catalytic core of Nei. Introduction of bulky fluorescent group between two specific sites greatly reduced the ability of glycosylases to slide along DNA. Acknowledgements: This work was supported by RSF (No. 14-24-00093). 